\title{
Maurice Lombard, Les Textiles musulmans du VIIe au
} XIIe siècle

Paris, Éditions de l'EHESS, 2002, 316 p. (bibliogr., cartes, index, graphiques) (coll. «Les réimpressions des éditions de l'EHESS »).

\section{Constant Hamès}

\section{(2) OpenEdition}

Journals

Édition électronique

URL : http://journals.openedition.org/assr/1270

DOI : 10.4000/assr. 1270

ISSN : $1777-5825$

Éditeur

Éditions de l'EHESS

Édition imprimée

Date de publication : 1 avril 2003

Pagination : 59-157

ISBN : 2-222-96732-5

ISSN : 0335-5985

Référence électronique

Constant Hamès, " Maurice Lombard, Les Textiles musulmans du VIle au XIle siècle ", Archives de sciences sociales des religions [En ligne], 122 I avril - juin 2003, document 122.34, mis en ligne le 18 novembre 2005, consulté le 21 septembre 2020. URL : http://journals.openedition.org/assr/1270 ; DOI : https://doi.org/10.4000/assr.1270 
d'une extrême subtilité quant au choix des séquences illustrées, les places relatives des différents personnages, les gestes et les objets rituels figurés, etc. l'auteur démontre la présence dans les images des intentions idéologiques mentionnées plus haut, tout en explicitant les procédés par lesquels le miniaturiste les a, à sa manière, exprimées, allant parfois jusqu'à s'écarter de la lettre du texte pour rendre plus sensible sa véritable portée. Cet essai constitue à mes yeux une véritable défense et illustration $\mathrm{du}$ renouveau des travaux historiques sur l'image et des hypothèses analytiques évoquées au début de cette note. Il mériterait pour cela seul d'être lu.

La dernière étude du recueil, celle de M.-N.C., porte sur la place du chant dans le sacre. Les notations très limitées de l'ordo étudié sont complétées par les informations recueillies dans les autres textes connus. On en retiendra en particulier avec l'auteur (p. 247), la place réservée aux références à l'Ancien Testament, bienvenues dès lors qu'est en question la royauté en terre chrétienne. L'ouvrage comporte enfin en appendice une étude de la langue et du style de l'ordo ainsi qu'une édition de l'intégralité du texte latin avec sa traduction, dues à Monique Goullet.

Jean-Pierre Albert.

Les Textiles musulmans du $\mathrm{VII}^{\mathrm{e}}$ au $\mathrm{XII}^{\mathrm{e}}$ siècle. Paris, Éditions de l'EHESS, 2002, 316 p. (bibliogr., cartes, index, graphiques) (coll. "Les réimpressions des éditions de l'EHESS $\gg)$.

Historien ( $6^{\mathrm{e}}$ section de l'EPHE) de l'économie et des techniques du monde musulman ancien, M.L. est décédé prématurément en 1964, sans avoir pu mettre en forme l'énorme quantité de matériaux et de références accumulée. Un ouvrage d'ensemble était cependant pratiquement rédigé et il fut édité, à titre posthume, en 1971: L'Islam dans sa première grandeur (VII $-X I^{e}$ siècles), aux éditions Flammarion. Ouvrage unique dans sa vision à la fois précise et globale des productions matérielles et des échanges dans le monde musulman et, en particulier, dans son analyse de la production et de la circulation monétaires.

Restait l'immense chantier consacré à des chapitres plus sectoriels de cette économie et qui débouchait, après mise en forme et, souvent, écriture, sur trois publications, consacrées respectivement à la monnaie (1971), aux métaux (1974) et aux textiles (1978) dont la réédition vient d'avoir lieu.

M.L. a eu le mérite et le courage de prendre le monde musulman pour ce qu'il était entre le $\mathrm{VIII}^{\mathrm{e}}$ et les $\mathrm{XI}^{\mathrm{e}}-\mathrm{XII}^{\mathrm{e}}$ siècles : une unité économique et civilisationnelle où le marchand, l'artisan et les cours politiques entretiennent les réseaux du commerce sur toutes les distances. L'unification musulmane s'est faite au détriment de vieilles barrières politiques, notamment sassanide et byzantine et à l'avantage d'une double ouverture vers l'Asie et vers l'Afrique du nord. Voilà le paysage à l'intérieur duquel l'auteur met en place ses investigations, dans le cas présent, sur les techniques de production des textiles, sur leur commercialisation et leur utilisation. Il rappelle d'abord que partout, les hommes de l'islam ont repris à leur compte, avant de les développer et de les démultiplier, les centres de production des régimes antérieurs ou concurrents. Ils ont ainsi repris l'exploitation de textiles déjà existants, comme la laine et le lin et en ont initié de nouveaux, surtout le coton et la soie, cette dernière reçue de la Chine, comme le papier, par l'intermédiaire de prisonniers chinois (bataille de Talas en 751). Dans la plupart de leurs emplois, les textiles passent par une phase de teinture et l'étude des produits végétaux, minéraux, simples ou composés, forme une partie extrêmement documentée de l'ouvrage. Mais, dans la mesure où, pour l'A., on est « autorisé à pleinement qualifier la civilisation musulmane de « civilisation du textile», est-il possible d'apprécier quantitativement les productions et la circulation de ces biens ? Démuni de statistiques, même discontinues, il s'engage, en guise de réponse, dans une analyse très détaillée des domaines d'usage des textiles: habillement avant tout, textile mobilier (tentures, tentes, tapis), textile de navigation (voiles) et, ce qu'on aurait tendance à oublier, le textile destiné à la fabrication du papier: essentiellement le lin (delta égyptien, Andalousie orientale) et puis le chanvre. Pour chacun de ces thèmes, aux vues d'ensemble succèdent des analyses régionales, souvent centre après centre, ce qui permet de construire des cartes précisant lieux de production et destinations commerciales, textile après textile. Ces cartes, en annexes et dans le texte, forment un outil incomparable d'utilisation de toutes les données des monographies. M. L. a particulièrement soigné, dans ses analyses, tout ce qui a trait au lexique: emprunts divers, transformations phonétiques, acculturations linguistiques et aboutissements postérieurs dans des langues non musulmanes.

La bibliographie impressionnante par sa diversité s'arrête en 1971, sinon avant. Mais, 
compte tenu du sujet et, comme le rappelle l'A., de la destruction à peu près complète de cette production ancienne extrêmement fragile, on peut considérer cette somme comme à peu près inaltérable.

L'analyse reste dans l'ensemble matérielle, technique et économique. Le religieux y affleure quelquefois, comme dans le cas de l'histoire du tissage de la somptueuse draperie (kiswa) qui recouvre la Ka'ba de la Mekke. Mais il restera à d'autres à employer cette base de données pour la relier à des pratiques de religion, comme dans le culte ou les rituels (tapis de prière, tapis des mosquées, linceuls, tissus des catafalques etc) ou dans l'habillement (l'habit de cotonnade en Afrique de l'islamisation était synonyme de passage à l'islam) ou dans l'ornementation, etc. Sur un plan historique plus général, on a parfois estimé que le travail de M.L. était une démonstration de la non-pertinence de la thèse de Henri Pirenne qui rendait l'islam responsable de la coupure de la Méditerranée entre Occident et Orient, dont l'empire nordique de Charlemagne aurait été une conséquence. Constatons simplement que M.L. ne s'écarte jamais de ses préoccupations économico-techniques et ne s'aventure pas dans l'histoire des constructions étatiques et de leurs avatars diplomatiques. Et lorsqu'il cite Pirenne, c'est à propos de « Draps de Frise ou draps de Flandre ?»...

\section{Constant Hamès.}

\subsection{5}

LUNEAU (René).

Comprendre l'Afrique. Évangile, modernité, mangeurs d'âmes. Paris, Karthala, 2002, 211 p. (bibliogr., index).

Sous ce titre généreux et ambitieux, le coauteur de La Terre Africaine et ses religions s'affronte aux défis de certains traits de la modernité religieuse africaine : le recours à la transe, l'omniprésence des prophètes et visionnaires, mais aussi le retour en force de la sorcellerie, du fétichisme ou des sacrifices rituels, des «pratiques » que la rumeur populaire et les médias contribuent largement et complaisamment à faire exister, en Afrique comme en Europe. L'Afrique que donne à comprendre R.L. c'est d'abord une Afrique catholique francophone qui peut se sentir menacée, au moins dans certains pays (la Côte d'Ivoire notamment), par l'expansion continue de l'islam mais aussi par l'agitation intempestive de l'Afrique protestante anglophone et de ses dérivés pentecôtistes et autres. Mais sur le plan anthropologique, la pierre d'achoppement sur laquelle bute toute une génération d'ethnologues et de missiologues reste l'ambiguïté des rapports qu'entretiennent ces chrétiens africains avec des croyances qui n'ont rien de «folklorique » puisqu'elles renouent avec les « peurs ancestrales», comme les «mangeurs d'âme», les «fusils nocturnes» ou les «coupeurs de sexe ». La question centrale de cet ouvrage est de savoir comment un processus culturel comme celui de la christianisation, engagé depuis plus d'un siècle et poursuivi à travers plusieurs générations, peut continuer à être mis en échec par un noyau de résistance aussi fort que celui qui entoure la croyance générale et partagée en la sorcellerie. Le défi n'est pas seulement cognitif (comment peut-on croire encore aux sorciers ?) interrogeant les effets en profondeur de la scolarisation et même de la culture scientifique et rationnelle transmise par les études universitaires sur les générations récentes, il est aussi et surtout éthique car comment admettre que des frères chrétiens puissent penser tout naturellement que les autres (les proches du village aussi bien que les étrangers) soient responsables de leurs malheurs et se transformer en bourreaux de leurs frères présumés sorciers (le spectre du génocide rwandais est en arrière-fond) ?

Un tel questionnement présuppose une complète extériorité du message chrétien par rapport à l'univers de croyances dit «traditionnel ». L'auteur n'ignore pas bien sûr le « malentendu originel » suscité et entretenu par la diabolisation missionnaire des génies païens et des esprits ancestraux et encore moins les formes de légitimation de la sorcellerie que représentent certaines pratiques charismatiques de délivrance mais il est clair qu'il s'agit là pour lui d'une trahison du message authentique de libération de l'Évangile. Il connaît également très bien toute la littérature $(\mathrm{P}$. Geschiere, J. Comaroff) sur la modernité religieuse et politique des imaginaires du mal, mais à ses yeux le parallèle que l'on peut tirer de la persistance au cœur des campagnes européennes des affaires de sorcellerie ou des apparitions miraculeuses reste l'argument décisif pour relativiser la vision d'une Afrique qui serait décidément vouée à l'irrationalité.

Tout le problème est que les situations de crise et de guerre civile de l'Afrique, le désengagement de l'État face aux problèmes d'éducation, de santé et de sécurité, n'en déplaise aux afro-optimistes, ne cessent d'augmenter le sentiment d'insécurité et de réveiller les « peurs ancestrales» qui rappellent les peurs de l'Occident. D'où le succès des formes de religiosité prophétique ou charismatique qui prétendent faire de Jésus, le «super-sorcier», la solution à tous les problèmes. Pour R.L. cette 